\title{
Which is better, EGFR-TKI mono or combination for non-small cell lung cancer with mutated EGFR?
}

\author{
Shigeru Tanzawa, Masashi Ishihara, Terunobu Haruyama, Ryosuke Ochiai, Takahiko Sakamoto, \\ Takeshi Honda, Shuji Ota, Yasuko Ichikawa, Kiyotaka Watanabe, Nobuhiko Seki \\ Division of Medical Oncology, Department of Internal Medicine, Teikyo University School of Medicine, Tokyo, Japan \\ Correspondence to: Nobuhiko Seki. Division of Medical Oncology, Department of Internal Medicine, Teikyo University School of Medicine, 2-11-1 \\ Kaga, Itabashi-Ku, Tokyo 173-8606, Japan. Email: nseki@med.teikyo-u.ac.jp. \\ Comment on: Saito H, Fukuhara T, Furuya N, et al. Erlotinib plus bevacizumab versus erlotinib alone in patients with EGFR-positive advanced non-squamous \\ non-small-cell lung cancer (NE7026): interim analysis of an open-label, randomised, multicentre, phase 3 trial. Lancet Oncol 2019;20:625-35.
}

Submitted Jul 31, 2019. Accepted for publication Aug 13, 2019.

doi: $10.21037 /$ tcr.2019.08.18

View this article at: http://dx.doi.org/10.21037/tcr.2019.08.18

Currently, three strategies for primary treatment of advanced non-small cell lung cancer (NSCLC) with EGFR gene mutations are being investigated: epidermal growth factor receptor (EGFR)-tyrosine kinase inhibitor (TKI) monotherapy, EGFR-TKI + vascular endothelial growth factor (VEGF) inhibitor combination, and EGFR-TKI + cytotoxic anticancer agent combination (Tables 1,2).

At the ASCO 2018 meeting, the results from a phase 3 study comparing erlotinib monotherapy for standard primary treatment versus erlotinib + bevacizumab combination were reported (NEJ026) (1). NEJ026 was based on a similarly designed phase 2 study (JO25567), which previously showed that erlotinib + bevacizumab combination significantly increased the primary endpoint, progression-free survival (PFS), compared with erlotinib monotherapy [16.4 vs. 9.8 months; hazard ratio (HR), 0.52; 95\% CI, 0.35-0.76] (2). Both were Japanese multicenter studies.

NEJ026 was an open-label randomized phase 3 study comparing erlotinib + bevacizumab combination with erlotinib monotherapy in 224 advanced-non-squamous NSCLC patients with EGFR gene mutations (Ex19 del/ Ex21 L858R) in performance status 0-2, with an interim analysis performed with PFS as the primary endpoint when 117 events had occurred. Since PFS (independent central judgment) was significantly higher in the combination group (16.9 vs. 13.3 months; HR, 0.61; 95\% CI, 0.42-0.88), the study was ended prematurely. By type of EGFR gene mutation, PFS was 16.6 vs. 12.4 months for Ex19 del (HR, $0.69 ; 95 \%$ CI, 0.41-1.16) and 17.4 vs. 13.7 months for Ex21 L858R (HR, 0.57; 95\% CI, 0.33-0.97): for both types of mutation, PFS was better in the combination group.
Reported adverse events (AEs) included hypertension (45.5\% vs. $8.8 \%$ ), proteinuria ( $32.1 \%$ vs. $2.6 \%$ ), and hemorrhage (25.0\% vs. $2.6 \%)$, which were all noted at higher incidences in the combination group than in the monotherapy group; however, the treatment discontinuation rate due to AEs was similar between the two groups (18.8\% vs. $15.2 \%)$. Overall survival (OS) data remain immature, with no final analysis results reported to date. Another subgroup analysis showed the PFS to be 16.9 vs. 12.6 months in the group with malignant pleural effusions (HR, 0.58 ; 95\% CI, $0.34-1.02)$ and 16.6 vs. 14.2 months in the group without (HR, 0.67; 95\% CI, 0.41-1.10): even in the population with pleural effusion, PFS increased in the combination group. In the group without cerebral metastases, PFS was higher in the combination group (18.0 vs .15.1 months; HR, 0.56; 95\% CI, 0.35-0.90). However, in the group with cerebral metastases, PFS did not differ between the two groups (12.7 vs. 11.2 months; HR, 0.78; 95\% CI, 0.42-1.43). As stated above, erlotinib + bevacizumab combination is advantageous not only with increased PFS for any type of EGFR gene mutation, but also with expected increases in PFS even in patients with pleural effusion; however, some issues remain to be resolved, including unknown OS-increasing effect, necessity for further investigations of effects on cerebral metastases, and definitely increased incidences of AEs.

Potentials of combinations of EGFR-TKI and VEGF inhibitors are discussed below in view of preclinical study results.

The first to discuss is a crosstalk between EGFR and VEGF, both key factors in tumor growth and metastases. When binding to EGFR, EGF and TGF- $\alpha$ activate EGFR to increase VEGF expressions. The crosstalk has 
Table 1 Clinical studies of EGFR-TKI in primary treatment

\begin{tabular}{|c|c|c|c|c|c|c|c|c|c|c|c|c|c|c|}
\hline Study & Investigational drugs & Phase & CNS mets & $\begin{array}{l}\text { Primary } \\
\text { endpoint }\end{array}$ & $\mathrm{N}$ & $\begin{array}{c}\text { PFS } \\
(\mathrm{m})\end{array}$ & HR & $\begin{array}{l}\text { OS } \\
(\mathrm{m})\end{array}$ & HR & $\begin{array}{c}\text { Ex19 } \\
\text { PFS } \\
(\mathrm{m})\end{array}$ & $\mathrm{HR}$ & $\begin{array}{c}\text { Ex21 } \\
\text { PFS } \\
(\mathrm{m})\end{array}$ & HR & $\begin{array}{c}\text { ORR } \\
(\%)\end{array}$ \\
\hline \multirow[t]{2}{*}{ NEJ002 } & Gefitinib & III & Included & PFS & 114 & 10.8 & 0.30 & 28 & 0.89 & & & & & 74 \\
\hline & CBDCA + PTX & & & & 114 & 5.4 & & 27 & & & & & & 31 \\
\hline WJOG3405 & CDDP + DOC & & & & 86 & 6.6 & & 37 & & & & & & 32 \\
\hline \multirow[t]{2}{*}{ OPTIMAL } & Erlotinib & III & Included & PFS & 82 & 13.7 & 0.16 & 23 & 1.19 & & & & & 83 \\
\hline & CBDCA + GEM & & & & 72 & 4.6 & & 27 & & & & & & 36 \\
\hline EURTAC & Erlotinib & III & Included & PFS & 86 & 9.7 & 0.37 & 23 & 0.92 & & & & & 61 \\
\hline LUXLUG3 & $\mathrm{CDDP}+\mathrm{PEM}$ & & & & 115 & 6.9 & & 28 & & & & & & 23 \\
\hline \multirow[t]{2}{*}{ LUXLUG6 } & Afatinib & III & Included & PFS & 242 & 11 & 0.28 & 23 & 0.93 & & & & & 74 \\
\hline & CDDP + GEM & & & & 122 & 5.6 & & 24 & & & & & & 31 \\
\hline \multirow[t]{2}{*}{ LUXLUG7 } & Afatinib & $\mathrm{Ilb}$ & Included & PFS, TTF, & 160 & 11 & 0.73 & 28 & 0.87 & 12.7 & 0.76 & 10.9 & 0.7 & 70 \\
\hline & Gefitinib & & & OS & 159 & 10.9 & & 25 & & 11 & & 10.8 & & 56 \\
\hline \multirow[t]{2}{*}{ FLAURA } & Osimertinib & III & Included & PFS & 279 & 18.9 & 0.46 & NR & 0.63 & 21.4 & 0.43 & 14.4 & 0.5 & 80 \\
\hline & Gefitinib/erlotinib & & & & 277 & 10.2 & & NR & & 11 & & 9.5 & & 76 \\
\hline \multirow[t]{2}{*}{ NEJ026 } & Erlotinib + bevacizumab & III & Included & PFS & 112 & 16.9 & 0.61 & NA & & 16.6 & 0.69 & 17.4 & 0.6 & 72 \\
\hline & Erlotinib & & & & 112 & 13.3 & & NA & & 12.4 & & 13.7 & & 66 \\
\hline \multirow[t]{2}{*}{ RELAY } & Erlotinib + ramucirumab & III & Excluded & PFS & 224 & 19.4 & 0.59 & NR & 0.83 & 19.6 & 0.65 & 19.4 & 0.6 & 76 \\
\hline & Erlotinib & & & & 225 & 2.4 & & NR & & 12.5 & & 11.2 & & 75 \\
\hline \multicolumn{15}{|c|}{ EGFR-TKI + chemotherapy } \\
\hline \multirow[t]{2}{*}{ NEJ009 } & CBDCA + PEM + gefitinib & III & Included & PFS, & 170 & 20.9 & 0.49 & 52 & 0.69 & & & & & 84 \\
\hline & Gefitinib & & & PFS2, OS & 172 & 11.2 & & 39 & & & & & & 67 \\
\hline \multirow[t]{2}{*}{ Noronha et al. } & CBDCA + PEM + gefitinib & III & Included & PFS & 174 & 16 & 0.51 & NR & 0.45 & & & & & 75 \\
\hline & Gefitinib & & & & 176 & 8 & & 17 & & & & & & 63 \\
\hline
\end{tabular}

CBDCA, carboplatin; CDDP, cisplatin; CNS mets, central nervous system metastases; Ex, type of EGFR gene mutation: Ex19 del or Ex21 L858R; EGFR, epidermal growth factor receptor; HR, hazard ratio; DOC, docetaxel; GEM, gemcitabine; NA, not assessed; NR, not reached; ORR, overall response rate; OS, overall survival; PEM, pemetrexed; PFS, progression-free survival; PTX, paclitaxel; TTF, time to treatment failure; TKI, tyrosine kinase inhibitor; VEGF, vascular endothelial growth factor. 
Table 2 Ongoing clinical studies of EGFR-TKI combinations in primary treatment

\begin{tabular}{|c|c|c|c|c|c|}
\hline Study & Investigational drugs & Phase & CNS mets & Primary endpoint & $\mathrm{N}$ \\
\hline \multirow[t]{2}{*}{ BEVERLY } & Erlotinib + bevacizumab & III & Excluded & PFS & 100 \\
\hline & Erlotinib & & & & 100 \\
\hline CTONG1509 & Erlotinib + bevacizumab & III & Included & PFS & 155 \\
\hline \multirow[t]{2}{*}{ TORG1833 } & Osimertinib + ramucirumab & II & Included & PFS & 60 \\
\hline & Osimertinib & & & & 60 \\
\hline \multirow[t]{2}{*}{ NCT03909334 } & Osimertinib + ramucirumab & ॥ & Included & PFS & 100 \\
\hline & Osimertinib & & & & 50 \\
\hline \multirow[t]{2}{*}{ WJOG9717L } & Osimertinib + bevacizumab & ॥ & Included & PFS & 60 \\
\hline & Osimertinib & & & & 60 \\
\hline \multicolumn{6}{|c|}{ EGFR-TKI + chemotherapy } \\
\hline NEJ032C/LOGIK1801 & CDDP/CBDCA + PEM + osimertinib & II & Included & Safety/ORR & 66 \\
\hline FLAURA2 & $\begin{array}{l}\text { Osimertinib + platinum doublet chemotherapy } \\
\text { Osimertinib }\end{array}$ & III & $\begin{array}{l}\text { Details still } \\
\text { Unknown }\end{array}$ & PFS & 556 \\
\hline
\end{tabular}

CBDCA, carboplatin; CDDP, cisplatin; CNS mets, central nervous system metastases; EGFR, epidermal growth factor receptor; ORR, overall response rate; PEM, pemetrexed; PFS, progression-free survival; TKI, tyrosine kinase inhibitor; VEGF, vascular endothelial growth factor.

been reported to occur between EGFR and VEGF in some forms, including non-EGFR-dependent increases in VEGF expression in resistance to EGFR inhibition. Experiments with a mouse xenograft model of human lung adenocarcinoma showed that increased expressions of the EGFR pathway due to gene expressional changes in interstitial cells, not in tumor cells, may be involved in the tumor resistance to VEGF inhibitors (3). It is reasonable to attempt to suppress tumor growth by blocking this EGFR-VEGF crosstalk with EGFR-TKI and VEGF inhibitors. In clinical settings, effects on malignant pleural effusion are expected. VEGF produced by tumor cells that have infiltrated the thoracic cavity is considered to act on the chest and diaphragm vascular endothelial cells to increase vascular permeability, resulting in the leakage of albumin and other plasma components from blood vessels, hence malignant pleural effusions (4). In fact, VEGF concentrations in malignant pleural effusions have been reported to be higher than exudative and leaking pleural effusions, which are caused by other factors (5), and some studies reported malignant pleural effusions to be controllable by bevacizumab combination chemotherapy in clinical settings. In study NEJ026 as well, a subgroup analysis by existence of pleural effusion showed that antitumor effects in patients with pleural effusion were expectable.

Second, we address effects on MET gene amplifications with resistance to EGFR-TKI. For the mechanism of acquisition of resistance to $1 \mathrm{st} / 2 \mathrm{nd}$-generation EGFRTKI, approximately $40-50 \%$ of patients have secondary mutations of $\mathrm{T} 790 \mathrm{M}$, and tumors acquire resistance to EGFR-TKI via many other mechanisms, including MET gene amplifications (up to $5 \%$ ) and transformation to small-cell carcinoma (4-10\%) (6). With regard to the mechanism of resistance acquisition in the patients in the osimertinib group, who experienced disease exacerbation during the FLAURA study, MET amplifications (15\%), EGFR C797S mutations (7\%), and other changes, along with T790M deletions, were noted (7). Preclinically, Furugaki et al. reported that a combination of erlotinib and bevacizumab was more effective than erlotinib or bevacizumab monotherapy in terms of tumor suppression 
in a cell line having both EGFR gene mutations and MET gene amplifications (8). Therefore, erlotinib + bevacizumab combination may be a treatment option for patients with MET amplification (9).

Third, potential effects on cerebral metastases should be taken into account. Generally, cerebral metastases in melanoma are of the perivascular progression type, whereas those in NSCLC are of the neovascularizationdependent type (10) and reportedly often increase vascular permeability and intensify cerebral edema (11). Therefore, bevacizumab combination is expected to be effective in treating or suppressing cerebral metastases. In fact, one study comparing CDDP+GEM therapy and CDDP + GEM + bevacizumab therapy for advanced lung adenocarcinoma reported that the incidence of cerebral metastases was lower in the bevacizumab combination group (AVAiL) (12). Another study found MET amplifications and/or MET protein expressions in $20-40 \%$ of the NSCLC patients with cerebral metastases (13); erlotinib+bevacizumab combination is expected to be effective in treating and suppressing cerebral metastases. In study NEJ026, however, no results were obtained to find any significance of bevacizumab combination in patients with cerebral metastases. One reason may be that tumor diameters are smaller in cerebral metastases in lung carcinoma with EGFR gene mutations (14). Hence, the patients with cerebral metastases enrolled in NEJ026 may have not enjoyed the best effects of VEGF inhibitors, because they had small tumor diameters in cerebral metastases and hence no severe cerebral edema. The NEJ026 results do not allow us to identify antitumor effects such as intracranial responses and PFS. A prospective confirmatory study comparing osimertinib monotherapy and osimertinib + bevacizumab combination in patients with cerebral metastases (NCT02971501) is ongoing and is expected to provide valuable data on their effects on central nervous system (CNS) lesions.

As stated above, combination with EGFR-TKI + VEGF inhibitors has much potential. Reported at the ASCO 2019 meeting was a phase 3 comparative study of erlotinib + ramucirumab combination compared with erlotinib monotherapy (RELAY). Erlotinib + ramucirumab combination significantly increased the primary endpoint, PFS, compared with standard erlotinib monotherapy (19.4 vs. 12.4 months; HR, 0.59; 95\% CI, 0.46-0.76) (15). As in study NEJ026, however, no OS results were reported. In the erlotinib + ramucirumab combination group, Grade $\geq 3$ hypertension was noted in $24 \%$ of the patients, and proteinuria, in $3 \%$; however, the proportion of patients who discontinued treatment because of AEs was nearly the same between the two groups. Although attention should be paid to increased incidences of hypertension and proteinuria with concomitant VEGF inhibitors, the two phase 3 studies (NEJ026, RELAY) suggest the toxicity of erlotinib + VEGF inhibitor combination to be tolerable.

Therefore, combination with EGFR-TKI and VEGF inhibitors represents a therapeutic strategy with great expectations for the future. Unless its superiority is finally demonstrated by OS results from the phase 3 study, however, it seems too early now to choose the regimen associated with increased incidences of AEs as the standard treatment. Currently, in addition to NEJ026, phase 3 studies of erlotinib + bevacizumab combination (BEVERLY, CTONG1509) are ongoing, attracting attention as to the reproducibility of their results. Although no significant difference was found in the OS for JO25567 reported at the ASCO 2018 meeting (47.0 vs. 47.4 months; HR, 0.81; 95\% CI, 0.53-1.23) (2), we await the publication of combined analysis results for OS from studies JO25567 and NEJ026.

On the other hand, a regimen is being developed with the EGFR-TKI drug to be combined with VEGF inhibitors for primary treatment switched from erlotinib to osimertinib. This is justified by the results from a phase 3 study comparing the $3 \mathrm{rd}$-generation drug osimertinib with the 1st-generation drug gefitinib/erlotinib (FLAURA) (16). The primary endpoint, PFS, increased significantly (18.9 vs. 10.2 months; HR, 0.46; 95\% CI, 0.37-0.57), and OS was better in the osimertinib group (HR, 0.63; $95 \%$ CI, 0.45-0.88), although the data were immature. The proportion of patients with Grade $\geqslant 3$ AEs was lower in the osimertinib group than in the gefitinib/erlotinib group, demonstrating better tolerability. Presented at the 2019 ASCO meeting were the results from a single-arm phase $1 / 2$ study of osimertinib + bevacizumab combination $(n=49)$, which reported overall response rate (ORR) of $80 \%$, PFS of 18.4 months, 1-year PFS rate of $70 \%$, and 1 -year OS rate of $91 \%$ (17). Randomized phase 2 studies comparing osimertinib monotherapy and osimertinib + bevacizumab combination (WJOG9171L, NCT02971501) and those comparing osimertinib monotherapy and osimertinib + ramucirumab combination (TORG1833, NCT03909334) are ongoing. Of the EGFR-TKI studies, osimertinib was found to be highly effective and tolerable, with a high CNS transfer rate: osimertinib + VEGF inhibitor combination is considered one of the most promising combination therapies.

In addition to the above EGFR-TKI + VEGF inhibitor combination, conventional EGFR-TKI monotherapy and EGFR-TKI + cytotoxic anticancer agent combination may also serve therapeutic strategies.

First, we'll discuss EGFR-TKI monotherapy. In a phase 3 study (ARCHER1050), dacomitinib, a 2nd-generation 
EGFR-TKI, increased not only PFS (14.7 vs. 9.2 months; HR, 0.59; 95\% CI, 0.47-0.74), but also OS (34.1 vs. 26.8 months; HR, 0.76; 95\% CI, 0.58-0.99), compared with gefitinib. However, the PFS in the dacomitinib group in ARCHER1050 was shorter than that in the osimertinib group in FLAURA. The incidence of Grade $\geqslant 3$ AEs was high at $48.9 \%$, posing toxicity management issues, including dose reductions and cessations (18). Therefore, osimertinib is currently used most commonly in primary treatment for lung carcinoma with EGFR gene mutations, since it surpasses 1st-generation EGFR-TKI in terms of both PFS and tolerability, and is also likely to surpass the 2ndgeneration drug dacomitinib in the same two aspects. There is room for argument, however, with regard to the PFS results by type of EGFR gene mutation in FLAURA. In the osimertinib group, the PFS was 21.4 months for Ex19 del and 14.4 months for Ex21 L858R. Better results were obtained from NEJ026, in which the PFS for Ex21 L858R with erlotinib + bevacizumab combination was 17.4 months, and from RELAY, in which the PFS for Ex21 L858R with erlotinib + ramucirumab combination was 19.4 months. Ex19 del and Ex21 L858R have so far been reported to also differ biologically (19). It seems it will become necessary in the future to use osimertinib monotherapy and EGFR-TKI + VEGF inhibitor combination in distinct ways according to the type of EGFR gene mutation.

Next to discuss is EGFR-TKI + chemotherapy combination. Reported at the ASCO 2018 meeting were the results of a phase 3 study comparing CBDCA + PEM + gefitinib combination with gefitinib monotherapy (NEJ009) (20). With PFS, PFS2, and OS as the primary endpoints, data were sequentially analyzed using a gatekeeping method. PFS differed significantly between the combination and monotherapy groups (20.9 vs. 11.2 months; HR, 0.49; 95\% CI, 0.39-0.63), whereas PFS2 did not differ significantly between the combination and monotherapy groups (20.9 vs. 20.7 months; HR, 0.97; 95\% CI, 0.77-1.22). This led to the publication of OS as a reference value despite the surprisingly longer survival in the combination group (52.2 vs. 38.8 months; HR, 0.70; 95\% CI, 0.52-0.93). At the ASCO 2019 meeting, a single study center in India published the results from a similar phase 3 study comparing gefitinib and CBDCA + PEM + gefitinib combination, which found significant differences in PFS between the combination and monotherapy groups (16 vs. 8 months; HR, 0.51; 95\% CI, 0.39-0.66) and in OS between the combination and monotherapy groups (OS not reached $v s .17$ months; HR, 0.45; 95\% CI, 0.31-0.65) (21). However, this result is difficult to accept universally, since the median OS for the monotherapy group was too short. Therefore, EGFR-TKI
+ cytotoxic anticancer agent combination remains to pose a challenging issue concerning OS, although it is considered an effective therapeutic strategy. In Japan, a single-arm phase 2 study of platinum + PEM + osimertinib for primary treatment in advanced-NSCLC patients with EGFR gene mutations (NEJ032C/LOGIK1801) is ongoing. Likewise, a global phase 3 study comparing platinum combination chemotherapy + osimertinib with osimertinib monotherapy for primary treatment (FLAURA2) is going to begin, with high expectation for favorable results (22).

Finally, the clinical implications of therapeutic strategies with EGFR-TKI monotherapy, EGFR-TKI+VEGF inhibitor combination, and EGFR-TKI + cytotoxic anticancer agent combination are discussed from the viewpoint of EGFR TKI-TKI sequence therapy. First, when osimertinib monotherapy is chosen as the primary treatment, no evidence is available for the strategy with other EGFR-TKI drugs administered after resistance development, making conventional cytotoxic anticancer agents the mainstay of secondary treatment. Although PFS2 was not reached in the osimertinib group in FLAURA, the lower limit of $95 \%$ CI was reported to be 23.7 months (23). When resistance developed with T790M mutations after treatment with 1st/2nd-generation EGFR-TKI, osimertinib increased PFS compared with cytotoxic anticancer agents (10.1 vs. 4.4 months; HR, 0.30; 95\% CI, 0.23-0.41) (AURA3) (24). Therefore, with erlotinib + bevacizumab and CBDCA + PEM + gefitinib combinations for primary treatment, PFS was 16.9 and 20.9 months, respectively, and PFS2 is expected to be approximately 27 and 31 months. However, the osimertinib use rate in the gefitinib/ erlotinib group in FLAURA was approximately 26\% (23). In a Japanese prospective observational study (REMEDY), the osimertinib use rate following resistance to $1 \mathrm{st} / 2 \mathrm{nd}$ generation EGFR-TKI was low at approximately $25 \%$ of the 236 patients in the full-analysis set (25). The strategy of administering osimertinib following treatment with 1 st/2ndgeneration EGFR-TKI still poses some problems to be solved, including difficulty with re-biopsy and T790M gene mutation detection rates.

In this editorial commentary, three patterns of promising treatment strategy for lung carcinoma with EGFR gene mutations were discussed: EGFR-TKI monotherapy, EGFR-TKI+VEGF inhibitor combination, and EGFRTKI + cytotoxic anticancer agent combination. Among them, EGFR-TKI+VEGF inhibitor combination is expected as a therapeutic strategy with potential clinical applications expected from the above preclinical study results. In reality, however, the advantages/disadvantages of the three strategies are difficult to compare on the basis 
of available clinical study results. This is because different types of EGFR-TKI are chosen for the three strategies: osimertinib often for monotherapy, erlotinib for VEGF inhibitor combination, and gefitinib for cytotoxic anticancer agent combination. Desirably, we will be able to establish the best strategy, based on comparisons of the efficacy and tolerability of osimertinib monotherapy vs osimertinib + VEGF inhibitor combination $v s$. osimertinib + platinum combination chemotherapy.

\section{Acknowledgments}

We would like to thank ASCA Corporation for editing and reviewing this manuscript for English language.

Funding: None.

\section{Footnote}

Provenance and Peer Review: This article was commissioned and reviewed by the Section Editor Dr. Wei Xu (Division of Respiratory Disease, Department of Geriatrics, the First Affiliated Hospital of Nanjing Medical University, Nanjing, China).

Conflicts of Interest: The authors have completed the ICMJE uniform disclosure form (available at http:// dx.doi.org/10.21037/tcr.2019.08.18). Dr. Tanzawa reports research fund from AstraZeneca, personal fees from Chugai Pharmaceutical, personal fees from BristolMyers Squibb, personal fees from MSD, outside the submitted work; Dr. Ota reports personal fees from Eizai, personal fees from Kyowa Kirin, personal fees from TAIHO PHARMACEUTICAL, outside the submitted work; Dr. Ichikawa reports personal fees from Chugai Pharmaceutical, personal fees from AstraZeneca, personal fees from Eisai, outside the submitted work; Dr. Watanabe reports personal fees from Chugai Pharmaceutical, personal fees from Novartis, personal fees from Pfizer, personal fees from MSD, personal fees from AstraZeneca, personal fees from GlaxoSmithKline, personal fees from Aflac, personal fees from GILEAD, personal fees from DAIICHI SANKYO, personal fees from Janssen Pharmaceutical, outside the submitted work; Dr. Seki reports personal fees and scholarship donation from Eisai, personal fees from MSD, personal fees and scholarship donation from ONO PHARMACEUTICAL, personal fees from Kyowa Kirin, personal fees and scholarship donation from DAIICHI SANKYO, personal fees and scholarship donation from TAIHO PHARMACEUTICAL, personal fees and scholarship donation from Chugai Pharmaceutical, personal fees from Novartis, personal fees from BristolMyers Squibb, personal fees, scholarship donation and Research fund from Boehringer Ingelheim, personal fees and scholarship donation from Merk, personal fees and scholarship donation from Eli Lilly, personal fees and scholarship donation from Pfizer, personal fees and scholarship donation from Takeda Pharmaceutical, personal fees from Bayer, personal fees and scholarship donation from SHIONOGI, personal fees from AstraZeneca, personal fees and scholarship donation from Nipponkayaku, outside the submitted work. The other authors have no conflicts of interest to declare.

Ethical Statement: The authors are accountable for all aspects of the work in ensuring that questions related to the accuracy or integrity of any part of the work are appropriately investigated and resolved.

Open Access Statement: This is an Open Access article distributed in accordance with the Creative Commons Attribution-NonCommercial-NoDerivs 4.0 International License (CC BY-NC-ND 4.0), which permits the noncommercial replication and distribution of the article with the strict proviso that no changes or edits are made and the original work is properly cited (including links to both the formal publication through the relevant DOI and the license). See: https://creativecommons.org/licenses/by-nc-nd/4.0/.

\section{References}

1. Saito H, Fukuhara T, Furuya N, et al. Erlotinib plus bevacizumab versus erlotinib alone in patients with EGFR-positive advanced non-squamous non-small-cell lung cancer (NEJ026): interim analysis of an open-label, randomised, multicentre, phase 3 trial. Lancet Oncol 2019;20:625-35.

2. Yamamoto N, Seto T, Nishio M, et al. Erlotinib plus bevacizumab (EB) versus erlotinib alone (E) as firstline treatment for advanced EGFR mutation-positive non-squamous non-small-cell lung cancer (NSCLC): Survival follow-up results of JO25567. J Clin Oncol 2018;36:abstr 9007.

3. Cascone T, Herynk MH, Xu L, et al. Upregulated stromal EGFR and vascular remodeling in mouse xenograft models of angiogenesis inhibitor-resistant human lung adenocarcinoma. J Clin Invest 2011;121:1313-28.

4. Yano S, Shinohara H, Herbst RS, et al. Production of experimental malignant pleural effusions is dependent on invasion of the pleura and expression of vascular 
endothelial growth factor/vascular permeability factor by human lung cancer cells. Am J Pathol 2000;157:1893-903.

5. Yanagawa H, Takeuchi E, Suzuki Y, et al. Vascular endothelial growth factor in malignant pleural effusion associated with lung cancer. Cancer Immunol Immunother 1999;48:396-400.

6. Sequist LV, Waltman BA, Dias-Santagata D, et al. Genotypic and histological evolution of lung cancers acquiring resistance to EGFR inhibitors. Sci Transl Med 2011;3:75ra26.

7. Ramalingam SS, Cheng Y, Zhou C, et al. LBA50 Mechanisms of acquired resistance to first-line osimertinib: preliminary data from the phase III FLAURA study. Ann Oncol 2018;29 Suppl 8:mdy424.063.

8. Furugaki K, Fukumura J, Iwai T, et al. Impact of bevacizumab in combination with erlotinib on EGFR-mutated non-small cell lung cancer xenograft models with T790M mutation or MET amplification. Int J Cancer 2016;138:1024-32.

9. Seki N, Natsume M, Ochiai R, et al. Promising Combination Therapy with Bevacizumab and Erlotinib in an EGFR-Mutated NSCLC Patient with MET Amplification Who Showed Intrinsic Resistance to Initial EGFR-TKI Therapy. Case Rep Oncol 2019;12:91-7.

10. Kienast Y, von Baumgarten L, Fuhrmann M, et al. Realtime imaging reveals the single steps of brain metastasis formation. Nat Med 2010;16:116-22.

11. Levin VA, Tonge PJ, Gallo JM, et al. CNS Anticancer Drug Discovery and Development Conference White Paper. Neuro Oncol 2015;17 Suppl 6:vi1-26.

12. Ilhan-Mutlu A, Osswald M, Liao Y, et al. Bevacizumab Prevents Brain Metastases Formation in Lung Adenocarcinoma. Mol Cancer Ther 2016;15:702-10.

13. Preusser M, Streubel B, Berghoff AS, et al. Amplification and overexpression of CMET is a common event in brain metastases of non-small cell lung cancer. Histopathology 2014;65:684-92.

14. Iuchi T, Shingyoji M, Itakura M, et al. Frequency of brain metastases in non-small-cell lung cancer, and their association with epidermal growth factor receptor mutations. Int J Clin Oncol 2015;20:674-9.

15. Nakagawa K, Garon EB, Seto T, et al. RELAY: A multinational, double-blind, randomized Phase 3 study of erlotinib (ERL) in combination with ramucirumab (RAM) or placebo (PL) in previously untreated patients with epidermal growth factor receptor mutationpositive (EGFRm) metastatic non-small cell lung cancer (NSCLC). J Clin Oncol 2019;37:abstr 9000.

16. Soria JC, Ohe Y, Vansteenkiste J, et al. Osimertinib in Untreated EGFR-Mutated Advanced Non-Small-Cell
Lung Cancer. N Engl J Med 2018;378:113-25.

17. Yu HA, Kim R, Makhnin A, et al. A phase 1/2 study of osimertinib and bevacizumab as initial treatment for patients with metastatic EGFR-mutant lung cancers. J Clin Oncol 2019;37:abstr 9086.

18. Wu YL, Cheng Y, Zhou X, et al. Dacomitinib versus gefitinib as first-line treatment for patients with EGFRmutation-positive non-small-cell lung cancer (ARCHER 1050): a randomised, open-label, phase 3 trial. Lancet Oncol 2017;18:1454-66.

19. Cho J, Chen L, Sangji N, et al. Cetuximab response of lung cancer-derived EGF receptor mutants is associated with asymmetric dimerization. Cancer Res 2013;73:6770-9.

20. Nakamura A, Inoue A, Morita S, et al. Phase III study comparing gefitinib monotherapy $(G)$ to combination therapy with gefitinib, carboplatin, and pemetrexed (GCP) for untreated patients (pts) with advanced nonsmall cell lung cancer (NSCLC) with EGFR mutations (NEJ009). J Clin Oncol 2018;36:abstr 9005.

21. Noronha V, Joshi A, Patil VM, et al. Phase III randomized trial comparing gefitinib to gefitinib with pemetrexed-carboplatin chemotherapy in patients with advanced untreated EGFR mutant non-small cell lung cancer (gef vs gef+C). J Clin Oncol 2019;37:abstr 9001.

22. Shepherd FA. Targeted Therapy: The New Frontier. Presented at 2019 ASCO Annual Meeting Education Session; May 31, 2019; Chicago, IL. Available online: https://meetinglibrary.asco.org/record/168046/video. Accessed July 26, 2019.

23. Planchard D, Boyer MJ, Lee JS, et al. Postprogression Outcomes for Osimertinib versus Standard-of-Care EGFR-TKI in Patients with Previously Untreated EGFR-mutated Advanced Non-Small Cell Lung Cancer. Clin Cancer Res 2019;25:2058-63.

24. Mok TS, Wu YL, Ahn MJ, et al. Osimertinib or Platinum-Pemetrexed in EGFR T790M-Positive Lung Cancer. N Engl J Med 2017;376:629-40.

25. Seto T, Nogami N, Yamamoto N, et al. Real-World EGFR T790M Testing in Advanced Non-Small-Cell Lung Cancer: A Prospective Observational Study in Japan. Oncol Ther 2018;6:203-15.

Cite this article as: Tanzawa $S$, Ishihara $M$, Haruyama $T$, Ochiai R, Sakamoto T, Honda T, Ota S, Ichikawa Y, Watanabe $\mathrm{K}$, Seki N. Which is better, EGFR-TKI mono or combination for non-small cell lung cancer with mutated EGFR? Transl Cancer Res 2019;8(6):2223-2229. doi: 10.21037/tcr.2019.08.18 